\title{
Anticonvulsants for Preventing Seizures in Patients with Chronic Subdural Haematoma: Pilot Study
}

\author{
Mohamed Adel Ghoneim*, Waleed Abbass \\ Department of Neurosurgery, Faculty of Medicine, Cairo University, Cairo, Egypt \\ Email: ${ }^{\star}$ madel1981@hotmail.com
}

How to cite this paper: Ghoneim, M.A. and Abbass, W. (2020) Anticonvulsants for Preventing Seizures in Patients with Chronic Subdural Haematoma: Pilot Study. Open Journal of Modern Neurosurgery, 10, 237-243. https://doi.org/10.4236/ojmn.2020.102025

Received: December 30, 2019

Accepted: March 14, 2020

Published: March 17, 2020

Copyright $\odot 2020$ by author(s) and Scientific Research Publishing Inc. This work is licensed under the Creative Commons Attribution International License (CC BY 4.0).

http://creativecommons.org/licenses/by/4.0/

\begin{abstract}
Introduction: There is increasing of the incidence of chronic subdural hematomas (CSDH), due to increase of the elderly population with increase of intake oral anticoagulation and antiplatelet drugs in this category of patients. Seizures occur as a complication in $2.3 \%$ to $20.4 \%$ of the patients. There is a considerable side effect associated with using of anti-epileptic drugs (AEDs). Aim of the Study: To rule the value of prophylactic antiepileptic drugs in prevention of seizures after surgical evacuation of chronic subdural hematoma. Methods: A prospective controlled non-randomized study was performed on 26 patients with chronic or subacute subdural hematoma that was surgically evacuated through burr-holes from April 2017 to June 2019. We divided the patients into 2 groups: group A patients received prophylactic antiepileptic drugs for 6 month and group B patients didn't receive prophylactic antiepileptic drugs and the 2 groups were observed to detect occurrence of post-operative seizures for 6-month follow-up after surgery. Medical history, demographic data and imaging characteristics were recorded. Results: Seizure prevalence for all patients during hospitalization and 6-month follow-up was zero among the 13 patients who received prophylactic anti-seizure medications, and was 1 (7.7\%) among the 13 patients who didn't receive prophylactic anti-seizure medications. Conclusions: The incidence of post-operative seizures in patients with chronic subdural hematoma operated upon by burr holes evacuation is low. The administration of prophylactic antiepileptic drugs decreases the incidence of seizures after evacuation of CSDH, but the difference was not significant. So prophylactic antiepileptic drugs should not be routinely given for all patients with CSDH operated upon by burr hole evacuation unless there is risk factor for seizure development.
\end{abstract}

\section{Keywords}

Chronic Subdural Hematoma, Seizure, Prophylaxis 


\section{Introduction}

Chronic subdural hematoma (CSDH) is a common disease in neurosurgical practices. There is increasing of the incidence of chronic subdural hematomas (CSDH), due to increase of the elderly population with increase of intake oral anticoagulation and antiplatelet drugs in this category of patients [1] [2]. Burr-hole surgery is the standard treatment performed with improvement of the symptoms, and good prognosis [3]. Seizures have been noted to develop as a complication in $2.3 \%$ to $20.4 \%$ of patients [4] [5]. There is a controversy in seizure prophylaxis and prevention of post-operative seizures following evacuation [6]. Due to their negative effect on quality of life, the prevention of seizures is important [7]. When seizures occur in patients with CSDHs, there is increase in the incidence of later seizures and the probability of secondary harm [5] [8]. The risk of seizure in CSDH increases in cases of alcoholic patients, history of stroke, and CT finding of atrophic change of the brain [9], at the time of surgery presence of organized hematoma or insertion of subdural drain associated with higher risk of seizure development [10].

This study is to rule the value of prophylactic antiepileptic drugs in prevention of seizures after surgical evacuation of chronic subdural hematoma.

\section{Patients and Methods}

A total of 26 patients with CSDH operated upon by burr hole evacuation surgery from January 2017 to June 2019.

History taken from patients included head trauma, risk factors of seizure like alcohol intake previous stroke, general and neurological examination of patients, laboratory investigation included coagulation profile, and CT brain.

Technique used:

All the patients had general anaesthesia, two separate linear skin incisions were done, two burr holes for the side if bilateral four bur holes were done, then the dura cauterized and opened, the subdural space irrigated by normal saline until fluid became clear, and then subgaleal drain was inserted for about 72 hours (no subdural drain used in our study).

The 26 patients with CSDH divided into two groups Group (A) 13 patients received prophylactic antiepileptic either Phenytoin (PHT) loading dose 15 $\mathrm{mg} / \mathrm{kg}$ injected slowly intravenously preoperative then followed by maintenance doses of $100 \mathrm{mg}$ orally or intravenously every 6 to 8 hours or levetiracetam with dose $500 \mathrm{mg} / 12$ hours orally or intravenously after evacuation of hematoma and for 6 month. Group (B) 13 patients didn't receive prophylactic antiepileptic drugs before or after evacuation of hematoma. Study include adult patients of both genders more than 18 years old, Chronic or subacute subdural hematoma. And exclude patients with CT showed acute subdural hematoma, Patients with previous history of seizures or presenting with seizures and any post-operative acute intraparenchymal hemorrhage, acute subdural hematoma, cerebral infarction, or subdural abscess. 
The observation period was from the date of the diagnosis of CSDH or the onset of symptoms of CSDH to 6 month after operation.

\section{Results}

In the Group A the study population consisted of 13 patients with a mean age of 60.3 years and a range from 30 to 81 years. There were 7 males $(53.8 \%)$ and 6 females (46.2\%) patients. A history of head trauma was obtained in 4 (30.8\%) patients, no history of alcohol intake, and history of stroke in 3 patients (23.1\%).

The Group B was consisted of 13 patients with a mean age of 63.5 years and a range from 48 to 84 years. There were 6 males (46.2\%) patients and 7 females (53.8\%). A history of head trauma was obtained in $4(30.8 \%)$ patients no history of alcohol intake, and history of stroke in 4 patients (30.8\%).

As regard clinical presentation in Group A the most common presenting symptom was headache in $38.5 \%$ of cases followed by weakness in $23.1 \%$, the median Glasgow Coma Score for Group A patients was 14.

In Group B the most common presenting symptom was headache in $46.2 \%$ of cases followed by weakness in $30.8 \%$, other presentations in $23 \%$. The median Glasgow Coma Score for Group B patients was 14.

As regard radiological appearance in Group A 12 (92.3\%) patients had chronic subdural hematoma (CSDH) while 1 (7.7\%) had subacute subdural hematoma, the average thickness was $13 \mathrm{~mm}$ and the average midline shift for Group A was $4.4 \mathrm{~mm}$. Atrophic brain changes in 5 patients (38.5\%).

Patients with chronic subdural hematoma (CSDH) included 8 patients with left sided CSDH, 3 patients with right sided CSDH and one patient with bilateral CSDH. Patients with subacute subdural hematoma (SASDH) included 1 patient with right sided SASDH.

In Group B 12 (92.3\%) patients had chronic subdural hematoma (CSDH) while 1 (7.7\%) had subacute subdural hematoma, patients with chronic subdural hematoma (CSDH) included 8 patients have left sided CSDH, 3 patients had right sided CSDH and one patient had bilateral CSDH. the average thickness was 13.7. The average midline shift was $4.5 \mathrm{~mm}$. Atrophic brain changes in 4 patients (30.8\%).

Surgery and Outcome in Group A All 13 patients underwent surgical evacuation by burr hole craniostomy. No organized hematoma at time of surgery. 12 patients received prophylactic antiepileptics in the form of phenytoin and one patient received levetiracetam. The administration of phenytoin was associated with one dermatological complication in the form of skin rash $(7.7 \%)$ that was managed conservatively with stoppage of phenytoin and shifting to levetiracetam. There was no mortality or seizure among this group of patients. In Group B All 13 patients underwent an operative procedure. Burr hole procedures were performed in all the 13 patients. No organized hematoma at time of surgery. There was no mortality or status epilepticus among this group of patients. 
As regard anticonvulsive Medication and Seizures in the Group A: Seizure prevalence for all patients during hospitalization and 6 month follow-up was zero among the 13 patients who received prophylactic anti-seizure medications. In the Group B: Seizure prevalence for all patients during hospitalization and follow-up was $1(7.7 \%)$ among the 13 patients who didn't receive prophylactic anti-seizure medications. CT brain was done for this patient with good result. The case that developed seizures was left sided chronic subdural hematoma.

Anticonvulsive Medication and Seizures in all of 26 patients seizure prevalence for all patients during hospitalization and follow-up was 1/26 (3.8\%). New seizure activity occurred in $1(7.7 \%)$ of the 13 patients who didn't receive prophylactic anti-seizure medications and none of the patients who receive prophylactic antiseizure medications. The patients who developed seizures 58 years male with left sided chronic subdural hematoma with no history of stroke in CT brain no atrophic changes, at time of surgery hematoma wasn't organized, and seizure activity wasn't associated with increased morbidity (Table 1).

\section{Discussion}

The incidence of seizures in patients with chronic subdural hematoma varied from $2.3 \%$ to $20.4 \%$ [4] [5]. This is higher than the numbers we found in daily clinical practice [11]. The causes of this high incidences and, varied numbers are not obvious. In our study the incidence of seizures in Group B not receiving antiepileptic was $7.7 \%$ and in two Groups were $3.8 \%$. In our study the mean age of two Groups 61.6 years with age ranges from 30 to 84 years. Similar to Flores et al.

Table 1. Demographical data, Clinical data, and radiological parameters.

\begin{tabular}{lcc}
\hline & Group A & Group B \\
\cline { 2 - 3 } & $\begin{array}{c}\text { Receive prophylactic } \\
\text { antiepileptic }\end{array}$ & $\begin{array}{c}\text { Didn't receive prophylactic } \\
\text { antiepileptic }\end{array}$ \\
\hline No. of patients & 13 & 13 \\
Mean age & 60.3 years & 63.5 years \\
Male & $7(53.8 \%)$ & $6(46.2 \%)$ \\
Female & $6(46.2 \%)$ & $7(53.8 \%)$ \\
Left side & 8 & 7 \\
Right side & 4 & 5 \\
Bilateral & $13 \mathrm{~mm}$ & 1 \\
Average thickness & $4.4 \mathrm{~mm}$ & 4.5 \\
Average midline shift & $5(38.5 \%)$ & $4(30.8 \%)$ \\
Atrophic change & 14 & 14 \\
GCS score & 4 patients (30.8\%) & 4 patients (30.8\%) \\
Head trauma & 3 patients (23.1\%) & 4 patients (30.8\%) \\
Pervious stroke & Headache (38.5\%) & Headache (46.2\%) \\
Most Common Symptom & Weakness (23.1\%) & Weakness (30.8\%) \\
\hline
\end{tabular}


[12], the mean age was 59 years (range: 18 - 98 years), and Huang et al. [13] mean age was 69.0 years and rang from (29 - 90 years). In our study in two Groups male equal to female and both of them were 13 patients in contrast to other studies in which male more than female. [12] [13] there is a history of head trauma in $30.8 \%$ of the patients. In contrast to other study in which $72 \%$ of patients had history of head trauma [13], the reason of that may be due to the minor trauma may pass without observation.

The incidence of seizures in Flores et al. [12] was low 2.3\% in spite he didn't give prophylactic antiepileptic in patients of chronic subdural hematoma treated by burr hole evacuation. He decided not to give prophylactic antiepileptic due to their side effect and the risk to develop seizures very low. Similar to results of Chen et al. [4], Ohno et al. [14] and Battaglia et al. [11], in contrast to that Sabo et al. [5] had notice that $32 \%$ of patients who didn't receive prophylactic antiepileptic developed seizure with high morbidity so they advice to give prophylactic antiepileptic. In our study, we prospectively analyzed 26 patients treated surgically for CSDH using a burr-hole and irrigation. The 26 patients were divided into two Groups, Group A 13 patients (50\%) received prophylactic antiepileptic drugs. In one patients phenytoin resulted in non-dangerous dermatological reaction and we shifted to levetiracetam. None of Group A had developed seizure. In contrast to that one patient of Group B (7.5\%) of the 13 patients who did not receive antiepileptic prophylaxis had develop seizure, not associated with addition in morbidity. This case had left sided chronic subdural hematoma, with no history of stroke in CT brain no atrophic change at time of surgery hematoma wasn't organized, without risk factors mentioned by Won et al. [9] in cases of alcoholic patients, history of stroke, and CT finding of atrophic change of the brain, and Yamada et al. [10] organized hematoma. Though the administration of prophylactic antiepileptic drugs prevents the development of postoperative seizure, the incidence of seizure in the other group was low and the difference was not significant. So, we recommend that antiepileptic drugs should not be given prophylactically for all patients with CSDH due to their side effect on old age patients. This is a pilot study provided for a larger large number of sample size in the future.

\section{Study Limitation}

Small sample which will be considered in the future.

\section{Conclusion}

The incidence of post-operative seizures in patients with chronic subdural hematoma operated upon by burr holes evacuation is low. The administration of prophylactic antiepileptic drugs decreases the incidence of seizures after evacuation of CSDH but the difference was not significant. So prophylactic antiepileptic drugs should not be routinely given for all patients with CSDH operated upon by burr hole evacuation unless there is risk factor for seizure development. 


\section{Conflicts of Interest}

The authors declare no conflicts of interest regarding the publication of this paper.

\section{References}

[1] Rust, T., Kiemer, N. and Erasmus, A. (2006) Chronic Subdural Haematomas and Anticoagulation or Anti-Thrombotic Therapy. Journal of Clinical Neuroscience, 13, 823-827. https://doi.org/10.1016/j.jocn.2004.12.013

[2] Kolias, A.G., Chari, A., Santarius, T. and Hutchinson, P.J. (2014) Chronic Subdural Haematoma: Modern Management and Emerging Therapies. Nature Reviews Neurology, 10, 570. https://doi.org/10.1038/nrneurol.2014.163

[3] Yamada, T. and Natori, Y. (2013) Risk Factors Associated with Recurrence after Burr-Hole Evacuation for Chronic Subdural Hematoma. Japanese Journal of Neurosurgery, 22, 125-132. https://doi.org/10.7887/jens.22.125

[4] Chen, C.W., Kuo, J.R., Lin, H.J., Yeh, C.H., Wong, B.S., Kao, C.H. and Chio, C.C. (2004) Early Postoperative Seizures after Burr-Hole Drainage for Chronic Subdural Hematoma: Correlation with Brain CT Findings. Journal of Clinical Neuroscience, 11, 706-709. https://doi.org/10.1016/j.jocn.2004.03.019

[5] Sabo, R.A., Hanigan, W.C. and Aldag, J.C. (1995) Chronic Subdural Hematomas and Seizures: The Role of Prophylactic Anticonvulsive Medication. Surgical Neurology, 43, 579-582. https://doi.org/10.1016/0090-3019(95)00155-7

[6] Grisoli, F., Graziani, N., Peragut, J.C., Vincentelli, F., Fabrizi, A.P., Caruso, G. and Bellard, S. (1988) Perioperative Lumbar Injection of Ringer's Lactate Solution in Chronic Subdural Hematomas: A Series of 100 Cases. Neurosurgery, 23, 616-621. https://doi.org/10.1227/00006123-198811000-00012

[7] Chang, E.F., Potts, M.B., Keles, G.E., Lamborn, K.R., Chang, S.M., Barbaro, N.M. and Berger, M.S. (2008) Seizure Characteristics and Control Following Resection in 332 Patients with Low-Grade Gliomas. Journal of Neurosurgery, 108, 227-235. https://doi.org/10.3171/JNS/2008/108/2/0227

[8] Van Breemen, M.S.M., Wilms, E.B. and Vecht, C.J. (2007) Epilepsy in Patients with Brain Tumours: Epidemiology, Mechanisms, and Management. The Lancet Neurology, 6, 421-430. https://doi.org/10.1016/S1474-4422(07)70103-5

[9] Won, S.Y., Konczalla, J., Dubinski, D., Cattani, A., Cuca, C., Seifert, V., Rosenow, F., Strzelczyk, A. and Freiman, T.M. (2017) A Systematic Review of Epileptic Seizures in Adults with Subdural Haematomas. Seizure, 45, 28-35. https://doi.org/10.1016/j.seizure.2016.11.017

[10] Yamada, T. and Natori, Y. (2012) Examination of Five Cases of Organized Chronic Subdural Hematoma. Neurosurgical Emergency, 17, 81-86.

[11] Battaglia, F., Lubrano, V., Ribeiro-Filho, T., Pradel, V. and Roche, P.H. (2012) Incidence and Clinical Impact of Seizures after Surgery for Chronic Subdural Haematoma. Neurochirurgie, 58, 230-234. https://doi.org/10.1016/j.neuchi.2012.04.002

[12] Flores, G., Vicenty, J.C. and Pastrana, E.A. (2017) Post-Operative Seizures after Burr Hole Evacuation of Chronic Subdural Hematomas: Is Prophylactic Anti-Epileptic Medication Needed? Acta Neurochirurgica (Wien), 159, 2033-2036. https://doi.org/10.1007/s00701-017-3298-6

[13] Huang, Y.H., Yang, T.M., Lin, Y.J., Tsai, N.W., Lin, W.C., Wang, H.C., Chang, W.N. and Lu, C.H. (2011) Risk Factors and Outcome of Seizures after Chronic 
Subdural Hematoma. Neurocritical Care, 14, 253-259.

https://doi.org/10.1007/s12028-011-9509-8

[14] Ohno, K., Maehara, T., Ichimura, K., Suzuki, R., Hirakawa, K. and Monma, S. (1993) Low Incidence of Seizures in Patients with Chronic Subdural Haematoma. Journal of Neurology, Neurosurgery, and Psychiatry, 56, 1231-1233. https://doi.org/10.1136/jnnp.56.11.1231 